\section{AB0701 ANTIBODY RAPID TEST POSITIVE HEALTH CARE WORKERS AT A GERMAN UNIVERSITY HOSPITAL: FIRST WAVE CHARACTERISTICS}

M. Beck ${ }^{1,2}$, A. Nieters ${ }^{2}$, M. Rizzi ${ }^{1}$, U. Salzer ${ }^{1}$, J. Thiel ${ }^{1}$, N. Venhoff ${ }^{1}$, N. Peter ${ }^{1}$, H. Eibel ${ }^{1}$, R. Voll ${ }^{1}, \underline{\text { S. Finzel }}{ }^{1}{ }^{1}$ Faculty of Medicine, University of Freiburg, Department of Rheumatology and Clinical Immunology, University Medical Centre, Freiburg im Breisgau, Germany; ${ }^{2}$ Institute of Immunodeficiency, Centre for Chronic Immunodeficiency, Freiburg im Breisgau, Germany

Background: Freiburg was among the most heavily affected German cities during the first wave of Sars-Cov-2 infections in spring 2020. Consequently, the University Medical Center Freiburg was one of the first hospitals in Germany to treat Covid19 patients.

Objectives: To assess the proportion and characteristics of health care workers (HCW) that have been infected during that first wave SARS-CoV-2 serum IgG and IgM antibodies were measured.

Methods: HCW ( $n=902$, mean age: 40.7 years) participated in this study, and filled out an epidemiological questionnaire. Serum samples were analysed for SARS-Cov-2 IgG/IgM antibodies via rapid diagnostic test (RT) and via ELISA. Statistical analyses were performed using STATA 14.2. An exposure prevention score was developed to quantify the adherence to preventive measures in everyday life.

Results: $902 \mathrm{HCW}$ were tested by RT, and 499 by ELISA. In total, $11.5 \%$ of recruited HCW were antibody-positive in the RT, $12.2 \%$ in the ELISA. $87.5 \%$ of RT positives, $98 \%$ of ELISA-positives reported symptoms, compared to $74.6 \%$ and $78 \%$ of negatives, respectively. Symptoms such as cough $(57 \% / 46 \%)$, loss of smell and taste $(34 \% / 5.2 \%)$, fatigue $(68 \% / 45 \%)$, fever $(48 \% / 24 \%)$, body aches $(45 \% / 22 \%)$, and headaches $(58 \% / 46 \%)$ were reported by significantly more RT positives compared to negatives. The respective differences were even more pronounced $(p<0.001)$ among ELISA-positives compared to negatives with $>50 \%$ of those positive reported impaired smell or taste compared to less than $7 \%$ among the group of ELISA-negatives $(p<0.00001)$.

In logistic regression models, shift work and belonging to the lowest quartile of the exposure prevention score were significantly associated with seropositivity in both tests. Exposure towards children was inversely associated with seropositivity, however, in the finally adjusted model only significant for those that were RT-positive, but not ELISA-positive, reflecting the lower specificity of the former. Conclusion: The endemic infection rate in HCW was high. HCW adhering to preventive measures in everyday life had lower infection rates.

Disclosure of Interests: Manuel Beck: None declared, Alexandra Nieters: None declared, Marta Rizzi: None declared, Ulrich Salzer: None declared, Jens Thiel Speakers bureau: BMS, Nils Venhoff Speakers bureau: Novartis, Nicole Peter: None declared, Hermann Eibel: None declared, Reinhard Voll Speakers bureau: Novartis, Grant/research support from: BMS, Pfizer, Novartis, Stephanie Finzel Speakers bureau: Novartis

DOI: 10.1136/annrheumdis-2021-eular.3780

\begin{tabular}{|l|l|}
\hline AB0702 & PITFALLS IN THE DIAGNOSIS OF COVID-19 \\
& - EXPERIENCES FROM A RHEUMATOLOGY \\
& OUTPATIENT CLINIC
\end{tabular}

S. G. Werner ${ }^{1}$, H. E. Langer ${ }^{1}$, P. Höhenrieder ${ }^{2}$, R. Chatelain ${ }^{2,3} .{ }^{1} R H I O$ (Rheumatology, Immunology, Osteology) Düsseldorf, RHIO Research Institute Düsseldorf, Düsseldorf, Germany; ${ }^{2}$ University of Witten-Herdecke, Faculty of Health, Witten, Germany; ${ }^{3}$ Evangelical Hospital Düsseldorf, Departement of Dermatology, Düsseldorf, Germany

Background: PCR (Polymerase Chain Reaction) is generally considered the gold standard for confirming the diagnosis in the early stages of SARS-CoV-2 (severe acute respiratory syndrome coronavirus 2) infection. However, in our rheumatology outpatient clinic we observed a significant discrepancy between clinical evidence of COVID-19 and PCR results.

Objectives: Aim of this retrospective study was to analyze the significance of PCR and serologic tests in the diagnosis of COVID-19 (Corona Virus Disease 2019) in a cohort of patients with rheumatic diseases.

Methods: Between March 2020 and January 2021, 35 patients with a history of established COVID-19 or typical signs and symptoms were identified on the occasion of a routine rheumatology follow-up examination in our institution. Previous diagnostic work-up in external facilities (results of PCR or antibody testing, imaging) was documented. Antibody ELISA-tests (IgG, IgA, IgM, Euroimmun) were performed in patients reporting typical signs and symptoms of COVID-19 in the past.

Results: PCR diagnostics had been performed in 15/35 patients (43\%), in 13/35 $(39 \%)$ at the onset of the first symptoms, in 2 subjects only 2 months later. PCR was positive in $7 / 13(54 \%)$ of those tested early, but negative in the two patients tested later. In 29/35 patients (83\%) SARS-CoV-2-ELISA tests were performed on the occasion of the routine rheumatologic examination (interval between first symptoms and testing on average 98 days, median86, range 4-283 days). In two of the initially negative individuals the second PCR was positive. ELISA tests were positive in all patients. SARS-CoV-2 IgM antibodies were positive in only two patients (however 55 and 71 days after disease onset), $n=8 / 29(28 \%) \lg G$ only, $n=9 / 29(31 \%) \lg G$ and $\lg A, n=12 / 29(41 \%) \lg A$ only. In these subjects, IgG antibodies did not develop even in the further course. Antibody titers were in part very high, but in part also very low (only just above the normal value), so even low titers were diagnostic obviously. In all patients with negative PCR, ELISA was positive and retrospectively led to confirmation of the diagnosis. Only in 13/35 patients $(37 \%)$ diagnosis had been made with the onset of the first symptoms or in the course of clinically manifest disease and had led to appropriate quarantine measures and contact tracing by the health authorities. In contrast, in the majority of patients $(63 \%)$, the diagnosis of COVID-19 infection was only made retrospectively on the occasion of a routine rheumatologic follow-up. However, 5 of these 22 patients (23\%) had quarantined themselves during the symptomatic phase. Titer histories were available from 12 patients. The titer became negative in 7 patients, after a mean of 188 days (median 202, min 51, max 296 days), and remained positive in 5 individuals (mean 190 days, median 191, min 122 , max 260 days). The change of the titer was independent of disease severity or antirheumatic therapy.

Conclusion: The results suggest that the importance of PCR in the diagnosis of COVID-19 may be overestimated. Therefore, antibody testing for SARS-CoV-2 should be performed in cases of clinical suspicion and negative PCR. In antibody diagnostics, special features were observed compared to other viruses, in particular, in some patients only low antibody titers or the absence of seroconversion with lack of development of $\lg G$ antibodies. Normalization of antibody titers in some patients supports the recommendation to vaccinate even after expired COVID-19 disease.

Disclosure of Interests: None declared

DOI: 10.1136/annrheumdis-2021-eular.3798

\section{$\mathrm{AB} 0703$ \\ THE COURSE OF COVID-19 INFECTION IN PATIENTS WITH ARTHRITIS RECEIVING TARGETED DMARDS}

E. Koltsova ${ }^{1,2}$, G. Lukina ${ }^{2}$, E. Shmidt ${ }^{3}$, K. Lytkina ${ }^{4}$, E. Rozochkina ${ }^{2}$,

E. Zhilyaev ${ }^{5,6}$ on behalf of MUAR. ${ }^{1}$ Research Institute of the Organization of Health and Healthcare Management, Rheumatology, Moscow, Russian Federation; ${ }^{2}$ Moscow Clinical Scientific Center, Rheumatology, Moscow, Russian Federation; ${ }^{3}$ City Clinical Hospital 1 named after N.I. Pirogov, Rheumatology, Moscow, Russian Federation; ${ }^{4}$ City Clinical Hospital\#4, Rheumatology, Moscow, Russian Federation; ${ }^{5}$ Russian Medical Academy of Continuing Professional Education, Rheumatology, Moscow, Russian Federation; ${ }^{6}$ CJSC European Medical Center, Rheumatology, Moscow, Russian Federation

Background: WHO declared the COVID-19 outbreak as a pandemic on March 12th, 2020. Assessing the risk of severe course in patients with rheumatic diseases, especially those who receive targeted immunosuppressive treatment, is an urgent problem for rheumatologists.

Objectives: determine the relationship between used targeted biologic and synthetic DMARDs (tDMARDs) and the severity of course of COVID-19 infection. Methods: The analysis included the data of patients with chronic arthritis and COVID-19, used tDMARDs. COVID-19 infection was confirm by serology tests or immune system molecules (immunoglobulins/antibodies). The presence of symptoms, the need for hospitalization, and the need for oxygen therapy were considered as indicators of the severity of the infection. We also analyzed the spread of the lung involvement according to CT data, symptoms during the disease (fever, cough, anosmia, diarrhea)

Results: Analyses included 78 patients, among them 32 patients has ankylosing spondylitis, 31 patients - rheumatoid arthritis, 12 patients has psoriatic arthritis, 3 patients - juvenile arthritis. The average age of the included patients was $51,8 \pm 11,69$. Most of patients used TNF inhibitors - 43 (Adalimumab -10, Golimumab -4, Infliximab - 4, Certolizumab pegol -3, Etanercept - 22), JAK inhibitor tofacitinib used 13 patients, 5 patients each was treated with Abatacept and Tocilizumab, 3 patients used Rituximab and 2 patients each used Netakimab and Ustekinumab (Table 1). None of the patients included in the analysis required treatment in the ICU and/or mechanical ventilation. The course of the disease in patients treated with tDMARDs did not seem to be more severe than in 5 patients with arthritis who stopped treatment with tDMARDs before the onset of the pandemic. There was a direct correlation between the severity of the infection and the age of the patients $(p=0.007)$ There were no significant differences in the severity of the infection depending on the drug, including when adjusting for age. 
Conclusion: COVID-19 does not look extremely dangerous in patients with rheumatic diseases, used target DMARDs. Used of tsDMARDs doesn't reliably increase the risk of COVID-19 severity. There is reliable correlation between age and COVID-19 severity.

Table 1. Cohort characteristics

\begin{tabular}{llllll}
\hline Drugs & Mean age & $\begin{array}{l}\text { Number of } \\
\text { patients }\end{array}$ & $\begin{array}{l}\text { Patients } \\
\text { with } \\
\text { symptoms }\end{array}$ & Hospitalized patients & $\begin{array}{l}\text { Asymptomatic } \\
\text { course }\end{array}$ \\
\hline Adalimumab & $41,0 \pm 11,4$ & 10 & 9 & 2 & 1 \\
Golimumab & $51,0 \pm 11,03$ & 4 & 4 & 0 & 0 \\
Infliximab & $42,7 \pm 11,8$ & 4 & 0 & 0 & 4 \\
Certolizumab & $41,6 \pm 11,7$ & 3 & 3 & 0 & 0 \\
Etanercept & $50,7 \pm 11,4$ & 22 & 20 & 6 & 2 \\
Tofacitinib & $56,8 \pm 10,4$ & 13 & 12 & 2 & 1 \\
Abatacept & $57,4 \pm 10,9$ & 5 & 4 & 1 & 2 \\
Tocilizumab & $48,8 \pm 10.9$ & 5 & 3 & 1 & 0 \\
Rituximab & $55,6 \pm 10,7$ & 3 & 3 & 1 & 0 \\
Netakimab & $44,0 \pm 15,8$ & 2 & 2 & 1 & 1 \\
Ustekinumab & $48,0 \pm 10,5$ & 2 & 1 & 0 & 1 \\
\hline
\end{tabular}

Disclosure of Interests: None declared

DOI: 10.1136/annrheumdis-2021-eular.3867

\begin{tabular}{l|l}
\hline AB0704 & TELEMEDICINE AT THE TIME OF COVID-19: THE \\
& EXPERIENCE WITH RA PATIENTS TREATED WITH \\
& JAK-INHIBITORS
\end{tabular}

C. Garufi ${ }^{1}$, F. R. Spinelli ${ }^{1}$, S. Mancuso ${ }^{1}$, F. Ceccarelli ${ }^{1}$, F. Conti ${ }^{1}{ }^{1}$ Sapienza University of Rome, Reumatologia, Dipartimento di Dipartimento di Scienze Cliniche Internistiche, Anestesiologiche e Cardiovascolari, Roma, Italy

Background: The spread of COVID-19, the lockdown, the limited access to care reevaluated the role of tele-consultation and self-assessment.

Objectives: Our aim was to evaluate in a cohort of Rheumatoid Arthritis (RA) patients treated with JAK-inhibitors (JAKi): the self-assessed disease activity during lockdown, the lockdown impact on fatigue, anxiety, depression and the prevalence of Covid-19.

Methods: We enrolled RA patients treated with baricitinib or tofacitinib. At baseline $(\mathrm{BL})$ and follow-up we collected: patients' demographic data, composite disease activity indices (CDAI, DAS28 ${ }_{\text {CRP }}$ ), global assessment (PGA), pain visual analogue scale (VAS), FACIT (functional assessment of chronic illness therapy) and a self-rating scale for disease impact on anxiety and depression (Zung-A/D). Patients were instructed on how to perform self-assessment through video-material and fulfilled the online form of "Rheumatoid Arthritis Impact of Disease" $(\mathrm{RAID})^{1}$ and "RA Disease Activity Index" (RADAI). To capture the pandemic effect, we compared patients in different status (remission, low, moderate and high-disease activity) at the last in-person visit (preCoV) through the DAS28 and CDAI, to the tele-health visit (THV), measured by the RAID. BL and pre-CoV ZUNG-A, ZUNG-D, FACIT questionnaires were compared with the online results during the pandemic. Exposure, tests and symptoms of Covid-19 were recorded. Data were expressed as mean \pm standard deviation or median (IQR) according to distribution.

Results: Twenty patients (median age $58.2 \pm 11.9$ and mean disease duration $153.5 \pm 112.7$ months) were treated with tofacitinib and 27 with baricitinib. The median time-lapse between the pre-CoV visit and the THV was 12 (IQR 4) weeks. DAS28CRP and CDAl significantly decreased from BL to pre-CoV visit. During the last in-person visit, 21 patients $(48.83 \%)$ were in remission, $9(20.93 \%)$ in low disease activity; according to the RAID, 15 (31.91\%) and 7 (14.89\%) patients were respectively in remission and low disease activity during the THV (Table A). PGA and pain significantly decreased from $B L$ to pre-Cov visit but worsened during the lockdown (Table A). FACIT remaining stable during THV. At THV, we detected a significant improvement of anxiety from $B L$ (Zung-A) and a tendency to lower depression scores compared to BL (Table A). JAKi showed a good safety profile considering Covid-19 symptoms, none of the patients was diagnosed with SarsCoV2 infection.

Conclusion: This is the first study on virtual assessment in RA patients treated with JAKi. The unique social experiment of the pandemic impaired the clinical response already achieved before the lockdown, without a collateral worseling of FACIT, anxiety and depression.

REFERENCES:

[1] Gossec L, et al. Ann Rheum Dis. 2009

[2] Stucki G, et al. Arthritis Rheum. 1995
Table A. DAS28, CDAI, RAID scores and patient-reported outcomes assessment at baseline and during the follow-up

\begin{tabular}{lcccc}
\hline & & BL & pre-CoV & THV \\
\hline DISEASE ACTIVITY & & $\mathbf{N}(\%)$ & $\mathbf{N}(\%)$ & $\mathbf{N}(\%)$ \\
\hline REMISSION & DAS28 & $0(0 \%)$ & $21(48.8 \%)$ & \\
& CDAI & $0(0 \%)$ & $10(22.7 \%)$ & $15(31.9 \%)$ \\
LOW DISEASE & RAID & & & \\
& DAS28 & $1(2.1 \%)$ & $9(20.9 \%)$ & \\
MODERATE & CDAI & $7(14.8 \%)$ & $23(52.2 \%)$ & $7(14.9 \%)$ \\
& RAID & & & \\
HIGH & DAS28 & $33(70.2 \%)$ & $12(27.9 \%)$ & $13(27.6 \%)$ \\
& CDAI & $17(37.1 \%)$ & $8(18.1 \%)$ & \\
GH & RAID & $13(27.6 \%)$ & $1(2.3 \%)$ & $12(25.5 \%)$ \\
Pain & DAS28 & $23(48.9 \%)$ & $3(6.8 \%)$ & $45(45)^{\star} \#$ \\
Zung A & CDAI & & & \\
Zung-D & RAID & $70(30)$ & $20(49.5)^{*}$ & $(48.5)^{\star} \#$ \\
FACIT & & $70(28)$ & $25(45.5)^{*}$ & $35(14)^{*}$ \\
& & $37(9)$ & $37(10.2)$ & $38(12)$ \\
\hline
\end{tabular}

${ }^{*} p \leq 0.001$ vs BL\# $p \leq 0.04$ vs preCoVData expressed as median (IQR)

Disclosure of Interests: Cristina Garufi: None declared, Francesca Romana Spinelli Speakers bureau: Abbvie, Eli Lilly, Consultant of: Gilead/Galapagos, Eli Lilly, Grant/research support from: Pfizer, Silvia Mancuso: None declared, Fulvia Ceccarelli: None declared, Fabrizio Conti Speakers bureau: Abbvie, Eli Lilly, Sanofi, Pfizer, Consultant of: Gilead/Galapagos

DOI: 10.1136/annrheumdis-2021-eular.3892

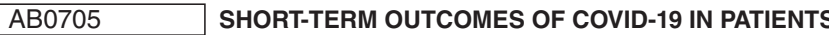 WITH RHEUMATIC DISEASES WHO ARE TREATED BY BIOLOGICAL AND TARGETED SYNTHETIC DMARDS: OBSERVATIONAL SINGLE-CENTER STUDY}

E. Luchikhina ${ }^{1}$, D. Karateev ${ }^{1}$, O. Semenova ${ }^{1}$, O. Matveychuk ${ }^{1}$, T. Kuznetsova ${ }^{1}$

${ }^{1}$ Moscow Regional Research and Clinical Institute n.a. M.F. Vladimirsky (MONIKI), Moscow Regional Rheumatology Center and Department of Rheumatology, Moscow, Russian Federation

Background: The course of new coronavirus infection in patients with rheumatic diseases $(\mathrm{RD})$ undergoing treatment with biological and targeted drugs is still poorly understood.

Objectives: To study outcomes of COVID-19 in patients with RD receiving treatment biological and targeted synthetic DMARDs.

Methods: We studied cases of COVID-19 in patients with RD, included in "Moscow regional registry of patients with rheumatic diseases receiving treatmen with biological and targeted synthetic drugs" - observational cohort, started in 2018. A total number of patients, included in the registry, is 1048 at December 2020.

Results: By January 2021, 44 known cases of COVID-19 were registered among patients included in the registry (4,2\%). This group included $29(65,9 \%)$ females, $15(34,1 \%$ ) males, with mean age 45,09 $\pm 12,7$ (median 47,0 [34,0; 57,0]) y.o. The vast majority of patients had rheumatoid arthritis $(19,43,2 \%)$ and ankylosing spondylitis $(19,43,2 \%)$, there were $3(6,8 \%)$ patients with psoriatic arthritis, and one patient each $(2,3 \%)$ with systemic lupus erythematosus, systemic sclerosis, and ANCA-vasculitis. Before COVID-19, 20 (45,5\%) patients received TNF inhibitors (adalimumab, infliximab, etanercept, certolizumab, golimumab) $7(15,9 \%)$ - IL-6 receptor inhibitors (tocilizumab, sarilumab), $7(15,9 \%)$ - ritux imab (period between last infusion and COVID-19 was 1-4 months), $5(11,4 \%)$ - sekukinumab, $2(4,5 \%)$ - tofacitinib, and one patient each $(2,3 \%)$ received abatacept and ustekinumab. Also, $22(50 \%)$ received methotrexate, $4(9,1 \%)$ - leflunomide, $3(6,8 \%)$ - mycophenolate mofetil, 1 (2,3\%) - sulfasalazine; 12 $(27,3 \%)$ took oral steroids. COVID-19 presented as mild disease in $23(52,3 \%)$ patients, and $21(47,7 \%)$ had viral interstitial pneumonia verified by computed tomography. $16(36,4 \%)$ patients were hospitalized, only one patient underwent artificial lung ventilation. We found no significant associations between particular diagnosis and treatment on the one hand, and hospitalization for COVID19 on the other hand. For treatment of COVID-19, two (4,5\%) patients did no receive any medications, and the rest of patients received antiviral and antibacterial therapy according to standardized protocol. In addition, corticosteroids were administered for COVID-19 in $15(34,1 \%)$ patients, mainly (12 cases) in hospital, and two $(4,5 \%)$ patients in hospital were treated by tocilizumab. The outcome in all cases was favorable, all patients successfully recovered from the new coronavirus infection. 